\section{OP0087 NATIONAL VARIATION AND FACTORS ASSOCIATED WITH THE TRANSITION FROM FIRST USE TO LONG- TERM OPIOID USE FOR NON-CANCER PAIN}

M. Jani ${ }^{1}$, B. Birlie Yimer ${ }^{1}$, T. Sheppard ${ }^{1}$, M. Lunt ${ }^{1}$, W. Dixon ${ }^{1} .{ }^{1}$ University of Manchester, Centre for Epidemiology Versus Arthritis, Manchester, United Kingdom

Background: Prescribing behaviour of physicians has been described as a key driver of rising opioid prescriptions and long-term opioid use. However, the effect of prescribers requires interpretation within context. No studies have investigated the extent to which regions, practices and prescribers, vary in opioid prescribing accounting for case-mix by considering this hierarchy together. Objectives: (i) Quantify and identify risk factors for the transition from newuser to long-term opioid user (ii) Quantify variation of long-term use attributed to region, practice and prescriber, accounting for patient mix and chance variation.

Methods: We conducted a retrospective observational UK study between 20062017 using Clinical Practice Research Datalink. Opioids new users, $\geq 18$ years, without cancer were identified. Long-term opioid use was defined as $\geq 3$ opioid prescriptions issued within a 90-day period from index date, or $\geq 1$ opioid prescription lasting at least 90 days in the first year. A multi-level random-effects logistic regression model was used to examine the association of patient characteristics with the odds of becoming a long-term opioid user. To examine variation in opioid use amongst prescribers, GP practices and region after adjusting for patient case-mix, we used a nested random-effect structure. A 'high-risk' region, prescriber or practice was defined as those where the entire adjusted $95 \% \mathrm{Cl}$ lay above the population average.

Results: $1,968,742$ new opioid users were included; $14.6 \%$ patients transitioned to long-term use. In the fully adjusted model, factors associated with higher odds of long-term opioid use included high morphine milligram equivalents (MME)/day at first prescription, older age, deprivation, fibromyalgia, rheumatological conditions, major surgery (Table). After adjustment for case-mix, the North-West, Yorkshire and South-West were found to be high-risk regions for long-term use. 103 practices $(25.6 \%)$ and 540 prescribers $(3.5 \%)$ were associated with a significantly higher risk of long-term use. The odds of becoming a long-term user for a patient belonging to these prescribers reached up to $>3.5$ times than the population average.

Conclusion: Prescribing factors, age, deprivation and conditions including fibromyalgia and rheumatological conditions were associated with higher odds of longterm opioid use. In the first UK study evaluating long-term opioid prescribing with adjustment for patient-level characteristics, variation in regions and especially practices and prescribers were observed. Our findings support greater calls for action to reduce practice and prescriber variation by promoting safe practice in opioid prescribing.

Table. Factors associated with long-term opioid use using a multi-level model accounting for clustering of individuals within prescriber, practice and region

Individual factors Adjusted Odds Ratio $(95 \% \mathrm{Cl})$

Prescribing factors

Index daily MME $>200$

Index daily MME 100-200

Index daily MME 50-100

Index daily MME $<50$

Gabapentinoid use

Psychotropic use

Age

$>75$
$65-75$
$55-65$

$55-65$

35-55

Age <35

Deprivation (Townsend score)

Quintile 5 (Most deprived)

Quintile 4

Quintile 3

Quintile 2

Quintile 1 (Least deprived)

Pre-existing conditions/ prior procedures

Fibromyalgia

Substance use disorde

Suicide and self-harm

Rheumatological conditions $\psi$

Alcohol abuse

Depression

Major Surgery

$7.59(6.29,9.16)$

$1.12(1.03,1.21)$

$1.58(1.49,1.68)$

Ref

$2.51(2.43,2.60)$

$1.28(1.17,1.40)$

$4.35(4.26,4.45)$

$3.57(3.50,3.65)$

$3.57(3.50,3.65)$
$3.03(2.96,3.09)$

$3.03(2.96,3.09)$
$1.91(1.88,1.95)$

Ref

$1.54(1.51,1.57)$

$1.34(1.31,1.36)$

$1.20(1.18,1.22)$

$1.09(1.07,1.11)$

Ref

$1.81(1.49,2.20)$

$1.76(1.70,1.83)$

$1.56(1.51,1.61)$

$1.54(1.49,1.59)$

$1.50(1.45,1.55)$

$1.28(1.26,1.30)$

$1.09(1.06,1.13)$

Abbreviations: MME, Morphine Milligram Equivalent; ${ }^{*} p<0.05$. Index daily MME/day is the $\mathrm{MME} /$ day at first prescription (MME = daily dose in milligrams $\mathrm{X}$ opioid conversion ratio). $\psi$ Defined by Charlson score including rheumatoid arthritis, SLE, myositis.
Disclosure of Interests: Meghna Jani Speakers bureau: Grifols, Belay Birlie Yimer: None declared, Therese Sheppard: None declared, Mark Lunt: None declared, William Dixon Consultant of: Bayer and Google DOI: 10.1136/annrheumdis-2020-eular.1725

OP0088

INITIATING TNF INHIBITORS IN INFLAMMATORY ARTHRITIS DOES NOT DECREASE THE AVERAGE OPIOID ANALGESIC CONSUMPTION

O. Palsson ${ }^{1,2,3,4}$, T. Love ${ }^{4,5}$, J. K. Wallman ${ }^{2,3}$, M. C. Kapetanovic ${ }^{2,3}$, P.

S. Gunnarsson ${ }^{6,7}$, B. Gudbjornsson ${ }^{1,4}$. $^{1}$ Landspitali University Hospital, Centre for Rheumatology Research, Reykjavik, Iceland; ' Lund University, Department of Clinical Sciences Lund, Rheumatology, Lund, Sweden;

${ }^{3}$ Skåne University Hospital, Department of Rheumatology, Lund, Sweden;

${ }^{4}$ University of Iceland, Faculty of Medicine, Reykjavik, Iceland; ${ }^{5}$ Landspitali University Hospital, Department of Science, Reykjavík, Iceland; ${ }^{6}$ University of Iceland, Faculty of Pharmaceutical Sciences, Reykjavik, Iceland;

${ }^{7}$ Landspitali University Hospital, Hospital Pharmacy, Reykjavik, lceland

Background: TNFa-inhibitor (TNFi) therapy is effective in controlling several rheumatic diseases and has been shown to reduce pain in patients with arthritis Opioids are often prescribed for chronic pain, a common issue in inflammatory joint disease.

Objectives: To explore the impact of the initiation of TNFi therapy as a firstline biologic disease-modifying anti-rheumatic drug (DMARD) on the prescription rates of opioids in patients with rheumatoid arthritis (RA), psoriatic arthritis (PSA), ankylosing spondylitis (AS) and undifferentiated arthritis (UA) in Iceland.

Methods: All patients receiving biologic DMARD therapy for rheumatic diseases in Iceland are registered in a nationwide database (ICEBIO). The Icelandic Directorate of Health operates a Prescription Medicines Register that includes over $90 \%$ of all drug prescriptions in Iceland. The study group included patients with RA, PsA, AS, and UA registered in ICEBIO and for each of them five randomly selected comparators from the general population matched on age, sex, and calendar time. On February $1^{\text {st }} 2016$ we extracted data on all filled opioid analgesic prescriptions two years before and two years after the date of TNFi initiation.

Results: Data from 359 RA, 217 AS, 251 PsA and 113 UA patients and 4700 comparators were collected. In total, $75 \%$ of patients compared to $43 \%$ of comparators received $\geq 1$ opiate prescription during the study period. The proportion of patients using opioids (regardless of dose) two years prior to TNF initiation was $41 \%$, increasing to $49 \%$ the following year. After TNFi initiation the proportion returned to $40 \%$ (Figure 1). Despite this, the mean yearly opiate dose used by the patients followed a rising trajectory throughout the study period (Figure 2). In total, patients were prescribed nearly 6 times more opioids than the comparators, corresponding to a bootstrapped mean $(95 \% \mathrm{Cl})$ dose of 818 (601-1073) mg MED per patient and year compared to 139 (111-171) mg for comparators.

Conclusion: Three out of four patients with inflammatory arthritis in Iceland use opioid analgesics in the two years prior to and/or after the initiation of TNFi therapy and the mean doses were significantly higher than in matched comparators. The proportion of patients receiving opioids increased before TNFi therapy and then decreased again to the previous level. The initiation of the first-line TNFi did not reduce opioid consumption by dose at the group level. On the contrary, there was a trend towards increasing doses over time in both patients and comparators, possibly reflecting the development of opiate tolerance.

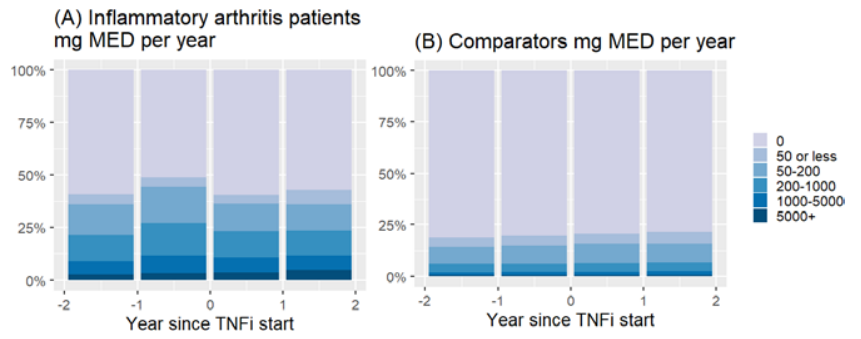

Figure 1. Percental distributions of opioid analgesic use by dose (according to dispensed prescriptions) among patients with inflammatory arthritis (A) and matched comparators (B). All doses are oral morphine equivalent dose (MED) in milligrams. 